\title{
Targeting T cell metabolism in the tumor microenvironment: an anti-cancer therapeutic strategy
}

\author{
Zhongping Yin ${ }^{\dagger}$, Ling Bai' ${ }^{\dagger}$, Wei Li, Tanlun Zeng, Huimin Tian and Jiuwei Cui
}

\begin{abstract}
T cells play important roles in anti-tumor immunity. Emerging evidence has revealed that distinct metabolic changes impact the activation and differentiation of T cells. Tailoring immune responses by manipulating cellular metabolic pathways and the identification of new targets may provide new options for cancer immunotherapy. In this review, we focus on recent advances in the metabolic reprogramming of different subtypes of $T$ cells and $T$ cell functions. We summarize how metabolic pathways accurately regulate T cell development, differentiation, and function in the tumor microenvironment. Because of the similar metabolism in activated T cells and tumor cells, we also describe the effect of the tumor microenvironment on T cell metabolism reprogramming, which may provide strategies for maximal anticancer effects and enhancing the immunity of T cells. Thus, studies of T lymphocyte metabolism can not only facilitate the basic research of immune metabolism, but also provide potential targets for drug development and new strategies for clinical treatment of cancer.
\end{abstract}

Keywords: T cell, Immunotherapy, Cancer, Metabolic reprogramming

\section{Background}

$\mathrm{T}$ cells are divided into many subtypes and kill tumors directly or indirectly by synthesizing various biological molecules. Naïve $T$ cells undergo metabolic reprogramming during proliferation, differentiation, and execution of effector functions. In recent years, studies of tumor and immune cell metabolism have shown that unlike resting cells, which mainly function in oxidative phosphorylation (OXPHOS), activated $\mathrm{T}$ cells mainly rely on aerobic glycolysis to obtain energy. Additionally, activated T cells can increase the decomposition of glutamine and reduce fatty acid oxidation (FAO) to meet the requirement of energy, cell growth, proliferation, differentiation, and cytokine secretion [1]. Therefore, different types of $\mathrm{T}$ cells are metabolically reprogrammed to perform their function.

Cancer cells also undergo metabolic reprogramming by upregulating glycolysis, glutamine decomposition, and lipid metabolism. These metabolic programs provide essential metabolites and energy for malignant proliferation,

\footnotetext{
*Correspondence: cuijw@jlu.edu.cn

${ }^{\dagger}$ Zhongping Yin and Ling Bai contributed equally to this work

Cancer Center, The First Hospital of Jilin University, Changchun 130021, Jilin, China
}

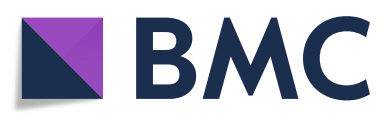

(c) The Author(s). 2019 Open Access This article is distributed under the terms of the Creative Commons Attribution 4.0 International License (http://creativecommons.org/licenses/by/4.0/), which permits unrestricted use, distribution, and reproduction in any medium, provided you give appropriate credit to the original author(s) and the source, provide a link to the Creative Commons license, and indicate if changes were made. The Creative Commons Public Domain Dedication waiver (http://creativecommons.org/publicdomain/zero/1.0/) applies to the data made available in this article, unless otherwise stated. invasion, metastasis, and adaptation to adverse living conditions [2]. Additionally, cancer cells regulate the differentiation of immune cells in the tumor microenvironment through their metabolites to indirectly promote cancer growth [3]. For example, tumor cell reprogramming inhibits effector T cells (Teffs) infiltration or induces apoptosis, promotes regulatory $\mathrm{T}$ cell (Tregs) differentiation, and exerts immunosuppressive functions by accumulating lactic acid, releasing carbon dioxide, etc. [4] Thus, understanding the regulation of tumor-induced metabolic stress on $\mathrm{T}$ cells are helpful to improve anticancer metabolic immunotherapy.

In this review, we summarize the latest advances in $\mathrm{T}$ cell metabolism and attempt to provide new ideas for anti-cancer therapy by targeting $\mathrm{T}$ cell metabolism. We also discuss targeted therapeutic measures for $\mathrm{T}$ cell metabolism through related pathways to further enhance the anti-cancer effect.

\section{Metabolic characteristics of $\mathrm{T}$ cell subtypes (Figure 1)}

Metabolism can be divided into two complex pathways: catabolic processes and anabolic processes. Catabolic 


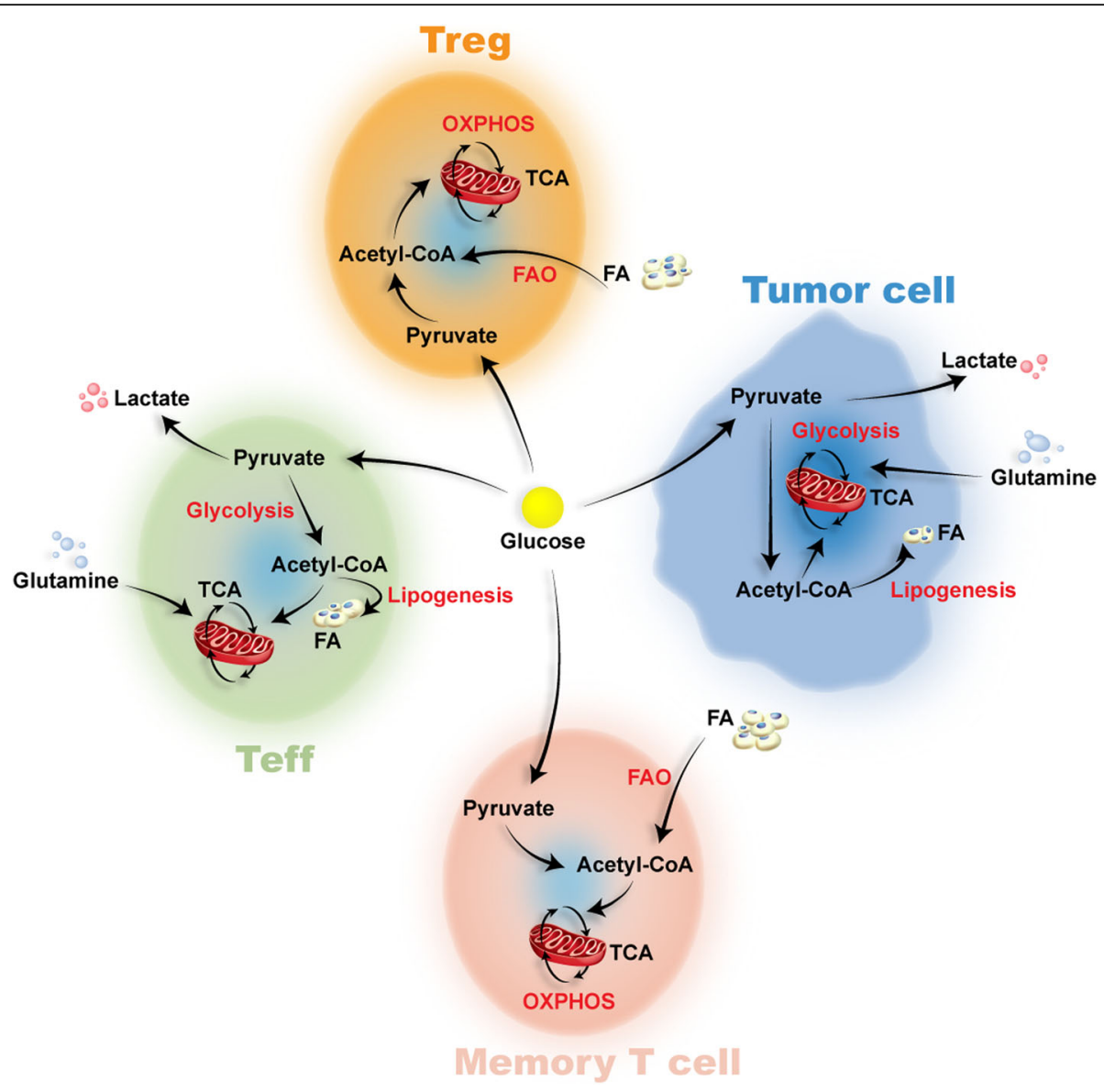

Fig. 1 Metabolic reprogramming of T cell subsets in tumor microenvironment. Tumor mainly use glycolysis to produce energy. Glucose competition, amino acid competition, and lactic acid secretion in the tumor microenvironment influence the subsets of $\mathrm{T}$ cells. Tregs mainly produce energy by oxidative phosphorylation (OXPHOS) and fatty acid oxidation (FAO). Similar to Treg, memory $T$ cells maintain basic functions by increasing FAO.

However, activated Teffs primarily depend on glycolysis and fatty acid synthesis

processes are critical for cellular proliferation and functions, while anabolic processes are important for cellular growth. Unlike cancer cells, $\mathrm{T}$ cell metabolic reprogramming is initiated by $\mathrm{T}$ cell receptor (TCR) recognition of antigens in the presence of costimulatory molecules. Transformation of this energy pattern contributes to the functions of rapidly proliferating $\mathrm{T}$ cells by providing the necessary raw materials and energy [5]. Besides, the integrity of mitochondria is also crucial for $\mathrm{T}$ cell function [6]. Thus, to better regulate immunity against the cancer microenvironment, an increasing number of studies have focused on the molecular mechanisms dictating metabolic reprogramming in different subtypes of $\mathrm{T}$ cells.

\section{Glucose metabolism}

$\mathrm{T}$ cells undergo metabolic reprogramming during activation, resulting in distinct functional fates [7]. Naïve T cells can generate ATP via OXPHOS. Because glycolysis can produce ATP faster and provide more nutrients for $\mathrm{T}$ cell activation than OXPHOS, $\mathrm{T}$ cell glucose metabolism occurs through glycolysis as observed in tumors to support their rapid growth and differentiation upon activation by the TCR and costimulatory signals [5]. During this process, naïve $T$ cells shift to Teffs, which can effectively kill tumor cells. In contrast, Tregs and memory $\mathrm{CD} 8^{+} \mathrm{T}$ cells rely on OXOPHOS for survival $[8,9]$. This metabolic pattern on memory $\mathrm{T}$ cells consumes large amounts of oxygen, which provides an energy basis for the rapid activation and effector function of the body after restimulation.

Because $\mathrm{T}$ cells at different stages have distinct demands for biological energy and biosynthesis, different signaling pathways are involved in the corresponding metabolic processes $[10,11]$. When $\mathrm{T}$ cells are activated, phosphoinositide 3-kinase (PI3K)/Akt signaling pathway activation can elevate mTOR signaling, increasing the expression of nutrient transporters (e.g., GLUT-1 or SLC1a5) and further promoting the glycolysis to improve the utilization rate of glucose [12]. Studies have shown that PI3K is related to p85 and p110 in glucose translocation. Akt is 
involved in regulating glucose metabolism through Forkhead box O1 (FOXO1) and glycogen synthase kinase-3. Additionally, AMP-activated protein kinase inhibited $\mathrm{T}$ cells activity by inhibiting mTORC1 activation. A recent study showed that the 6-phosphofructo-2-kinase/fructose2,6-biphosphatase 3 (PFKFB3) gene is over-expressed in immune cells and increases concomitantly with glucose transporter-1 (GLUT-1), hexokinase-II, and proliferating cell nuclear antigen upregulation, demonstrating that induction of T-cell proliferation by mitotic agents is required for metabolic reprogramming. PI3K/Akt pathway inhibitors (e.g., Akti-1/2 and LY294002) can reduce PFKFB3 gene induction by phytohemagglutinin, as well as fructose-2,6-bisphosphate and lactate production [13]. Thus, the substances that affect the activation of PI3K/Akt signaling pathway may affect the glycolysis of $\mathrm{T}$ cells. For example, Acylglycerol kinase, as a specific lipid kinase, can induce the phosphorylation of PTEN, thereby inactivating PTEN and maintaining metabolism and function of $\mathrm{CD}^{+}$ $\mathrm{T}$ cells [14]. Additionally, mTOR signaling pathway also regulates Treg glucose metabolism. A study has found that TCR activation promotes the assembly and activation of the mTORC1 complex of Treg on lysosomal membrane, while TRAF3IP3, a transmembrane molecule located on lysosomal membrane, can inhibit mTORC1 activity and its mediated glycolysis level. Thus, TRAF3IP3 maintains the stability and function of Treg [9]. Besides, epigenetic regulation is also important for the activation of signaling pathways. Just as deubiquitinating enzyme Otub1 can regulates $\mathrm{T}$ cell activation via inhibiting the activation of ubiquitin-dependent Akt [15].

In addition to the PI3K/Akt/mTOR signaling pathway, the transcription factor C-MYC-related pathway and nuclear receptor family pathway play important roles in glucose metabolism in T cells. C-MYC can enhance glycolysis by up-regulating the expression of GLUT-1 in activated $\mathrm{T}$ cells. As the key factor regulating the metabolic pathway to adapt to the requirements of $\mathrm{T}$ cells during activation, $\mathrm{T}$ cells without $\mathrm{C}-\mathrm{MYC}$ cannot survive and differentiate [16].

Recent studies have also focused on nutrient transporters and enzymes related to glucose metabolism. For example, miR-143 regulates $\mathrm{T}$ cell differentiation by inhibiting GLUT-1 [17]. Autophagy related gene Atg5 can regulate the change of histone methylation, inhibit the metabolism and upregulation of the transcription of effector target genes (such as GLUT-1), thus inhibiting the glucose metabolism of $\mathrm{CD}^{+} \mathrm{T}$ cells and interferon (IFN)- $\gamma$ secretion [18]. In studies on glycometabolism-related kinases, it was found that damage to glycolyze-related enzymes could inhibit $\mathrm{CD}^{+} \mathrm{T}$ cell function, such as ENOLASE 1 [19]. Acetate, a metabolite, enhances IFN-rproduction in exhausted T cells with an acetyl-CoA synthetase-dependent manner under low-glucose conditions [20].
Furthermore, memory $\mathrm{T}$ cells in pleural effusion secondary to lung cancer cannot upregulate CD71 and GLUT-1 when activated under hypoxic conditions, and the glycolysis is defective [21]. Hence, the metabolic changes of $\mathrm{T}$ cells in special cases are worthy of attention.

\section{Lipid metabolism}

Lipid metabolism mainly includes fatty acid metabolism and cholesterol metabolism. Under hypoxic conditions, hypoxia-inducible factor (HIF)- $1 \alpha$ induces pyruvate to leave mitochondria with OXPHOS, making Tregs dependent on fatty acids for mitochondrial metabolism in hypoxic tumors. Thus, FAO is crucial for Treg metabolism in cancer [22]. Besides, Teffs can obtain fatty acids for the microenvironment, while memory $\mathrm{T}$ cells only use carbon derived from glucose metabolism to synthesize fatty acids [23]. And lipid metabolism is also important for maintaining the balance between Teffs and Tregs [24].

Fatty acid synthesis (FAS) is mainly used to produce key lipid cell structures such as cell membrane necessary for cell proliferation, while FAO mainly provides ATP for cells and produces many metabolic intermediates with important physiological functions. During the synthesis of fatty acids, sterol regulatory element-binding protein (SERBP)-1 is activated by PI3K/Akt signaling pathway, and then ATP citrate lyase (ACLY) and fatty acid synthase (FASN) are up-regulated to promote the synthesis of fatty acids [25]. Activated T cells mainly rely on FAS [26], while naïve $\mathrm{T}$ cells and memory $\mathrm{T}$ cells maintain basic functions such as membrane functional integrity by increasing FAO [8]. FAO can inhibit the activation of Teffs by increasing programmed cell death protein 1 (PD-1) expression, promoting carnitine palmitoyltransferase $1 \mathrm{~A}$, one of the rate-limiting enzymes of FAO, and inhibiting IFN- $\gamma$ secretion. While FAO can promote Treg cells generation through MAPK signaling pathway activation [27]. Besides, Tregs are important for immune homeostasis. By promoting SERBP-1-dependent lipid metabolism, Treg cells inhibit $\mathrm{CD}^{+} \mathrm{T}$ cells to produce IFN- $\gamma$, maintain the immunosuppression of tumor-related macrophages, and coordinate the tumorrelated immunosuppression microenvironment [28]. Peroxisome proliferator-activated receptors (PPARs) can also regulate lipid metabolism, and its high activation is associated with immunosuppression. Increased PPAR- $\gamma$ activity can inhibit lipolysis, limit $\mathrm{T}$ cells OXPHOS, and promote the differentiation of Tregs [29].

Cholesterol can participate in maintaining cell membrane homeostasis and is a synthetic raw material for vitamin $\mathrm{D}$, bile acids, and steroid hormones. In-depth studies showed that the metabolism of immune cells in the tumor microenvironment was affected by the changes of the cholesterol. When $\mathrm{T}$ cells are activated, TCR activation promotes cholesterol synthesis by 
affecting the transcription of key enzymes in the cholesterol biosynthesis pathway (CBP). Meanwhile, liver $\mathrm{X}$ receptor (LXR) [30], SERBP-2, and acyl-CoA acyltransferase (ACAT)-1 play key regulatory roles in maintaining intracellular cholesterol stability [31, 32]. Early studies showed that the cholesterol levels of both whole cells and plasma membrane were markedly increased in activated $\mathrm{CD}^{+} \mathrm{T}$ cells. When $\mathrm{T}$ cells are activated, lipid mediators and cytokines promote $\mathrm{T}$-cell migration, proliferation, and differentiation [11, 33-36]. ACAT-1 and ACAT-2 are two key genes encoding cholesterol esterification enzymes that convert free cholesterol to cholesteryl esters for storage. ACAT-1 was mainly expressed in $\mathrm{CD}^{+} \mathrm{T}$ cells. Upon $\mathrm{CD}^{+} \mathrm{T}$ cell activation, ACAT- 1 was up-regulated in an early stage. Additionally, ACAT-1 deficiency inhibits cholesterol esterification but promotes biosynthesis of cholesterol, which can upregulate the cholesterol level in the plasma membrane of $\mathrm{CD}^{+} \mathrm{T}$ cells, eventually enhancing TCR clustering and signaling as well as the resulting in the more efficient formation of the immunological synapse [37]. However, recent studies found that cholesterol or its derivatives, through LXR Sumoylation, can reduce the binding of P65 to the IL-9 promoter and further inhibit the expression of IL-9, thereby inhibiting Tc9 cells differentiation and its anti-cancer response [38]. Given the contradictions in the role of intracellular cholesterol, the researchers found that high cholesterol in tumor-infiltrating lymphocytes (TILs) upregulate the expression of XBP1, an endoplasmic reticulum stress receptor, which further promotes the expression of the immune checkpoint and inhibit $\mathrm{T}$ cell function [39]. TILs, unlike $\mathrm{CD} 8^{+}$ $\mathrm{T}$ cells cultured in vitro, are generally limited in their antitumor activity due to their expression of many inhibitory receptors [40]. Thus, studies on the effect of intracellular cholesterol on $\mathrm{T}$ cell function need to focus on the $\mathrm{T}$ cell types.

\section{Amino acid metabolism}

In addition to glucose and lipid, cell growth and function are also dependent on amino acids. Apart from nucleotides and protein synthesis, amino acids participate in a variety of metabolic pathways.

The influx of branched chain amino acids (such as leucine and glutamine) is critical for Teff cell differentiation and function through mTORC1 activation. Glutamine or leucine expression levels can affect the activation and function of $\mathrm{T}$ cells. For example, down-regulation of glutamine and leucine metabolism has been shown to inhibit the differentiation of TH1 and TH17 effector T cells while maintaining Treg differentiation. When T cells are activated, key amino acid transporters can be up-regulated through the activation of metabolic regulators such as C-MYC. Additionally, glutamine is converted to glutamate by glutaminase. Decreased glutamine and leucine metabolism will reduce mTORC1 activity and C-MYC expression, resulting in blocked $\mathrm{T}$ cell activation [41]. This reduction in glutamate metabolism causes immune cells to develop into Treg cells. Meanwhile, glutaminase can enhance IL-2-mediated mTORC1 signaling pathway activation to promote TH17 differentiation and inhibit $\mathrm{TH} 1$ as well as cytotoxic lymphocyte (CTL) differentiation [42].

Similar to the secondary pleural effusion of lung cancer, ovarian malignant ascites can downregulate the GLUT-1 expression levels on $\mathrm{CD} 4^{+} \mathrm{T}$ cells, which leads to the defection of $N$-linked protein glycosylation, thereby promoting IRE1 $\alpha$-XBP1 activation. The activation of XBP1 regulates the glutamine transporters expression and further restrict the inflow of glutamine under glucose deprivation conditions, thereby inhibiting $\mathrm{T}$ cell infiltration and IFN- $\gamma$ secretion [43].

\section{Effects of tumor metabolism on $\mathrm{T}$ cells}

Tumor mainly use glycolysis to produce energy, which causes the microenvironment to become acidic and hypoxic; some metabolic intermediates can impair the antitumor effect of Teffs. Glucose competition, amino acid competition, oxygen competition, and lactic acid secretion in the tumor microenvironment promote formation of the immunosuppressive phenotype. Therefore, determining the influence of the tumor microenvironment on $\mathrm{T}$ cell metabolism will be helpful for developing methods to enhance the anti-tumor effect of $\mathrm{T}$ cells while also killing cancer cells.

\section{Tumor metabolism reprogramming indirectly regulates tumor microenvironment}

Tumor cells have infinite proliferation potential, in contrast to normal cells. To meet the demand for unlimited proliferation, tumor cells alter their metabolic patterns in glucose metabolism. An increasing number of studies has shown that tumors are not a homogeneous mass of malignant cells, but rather a complex structure containing vascular and stromal cells that support the tumor as well as a diverse array of infiltrating immune cells including lymphocytes and myeloid-derived cells. These cells alter their metabolic mode to proliferate in their specific environment. This adaptation which involves energy metabolism changes in the tumor is known as metabolic reprogramming. Normally, tumor cells mainly use glycolysis to provide ATP for rapid growth and use glutamine, lipids, and other substances to promote proliferation [44, 45]. Tumor cells also consume large amounts of oxygen and amino acids in the process of metabolism. During tumor progression, changes in some metabolites mediate the changes in immunomodulatory molecules, which are important factors leading to immune escape. Additionally, tumor cells compete with 
immune cells in the microenvironment for the components required for their own metabolism, further inhibiting immune cell functions.

Therefore, tumor metabolic reprogramming promotes tumor cell growth, with the resulting metabolites indirectly regulating the tumor microenvironment and ensuring tumor progression.

\section{Lactic acid in the tumor environment affects T cell function}

Warburg glycolysis allows cancer cells to consume glucose and increase lactic acid, glutamine, and $\mathrm{CO}_{2}$ production, resulting in acidification of the tumor microenvironment. The consumption of these metabolic substrates also negatively affects the high-metabolism of $\mathrm{T}$ cells. Lactic acid and other metabolites produced through these metabolic processes also inhibit the proliferation and function of $\mathrm{T}$ cells to varying degrees [46]. Lactic acid, as a glycolysis product, can inhibit the PI3K/ Akt/mTOR pathway and thus inhibit $\mathrm{T}$ cell glycolysis $[47,48]$. Additionally, acidification of the tumor microenvironment impairs Teffs to a much greater extent compared to in Tregs, mainly because Teffs acquire energy mainly through glycolysis, while Tregs can rely on fatty acid oxidation. Lactic acid and an acidic tumor microenvironment can promote high levels of cytokines secretion, monocarboxylate transporter 1 inhibition, and thus promote neovascularization as well as glycolytic flux decreation[49, 50]. Meanwhile, the decrease of $\mathrm{pH}$ in the tumor microenvironment not only increases the infiltration of $\mathrm{CD}^{+} \mathrm{T}$ cells and NK cells but also reduces the number of Tregs to maintain the immunosuppressive tumor microenvironment [51].

\section{Hypoxia in the tumor environment affect $T$ cell function}

Because tumor aerobic glycolysis consumes a large amount of oxygen, the tumor microenvironment is often anoxic. A study conducted at the Weizmann Institute of Science found that hypoxic cultured $\mathrm{T}$ cells killed tumor cells by releasing high levels of granzyme $B$, a destructive enzyme, but not perforin. Adoptive treatment with hypoxic $\mathrm{T}$ cells increases the survival time of mice with OVA-expressing B16 melanoma cells [52].

HIF- $1 \alpha$ is the main transcriptional regulator in the cellular response to hypoxia and is the downstream target of GLUT-1, which facilitates glucose uptake. Under hypoxic conditions, high HIF-1 $\alpha$ expression leads to loss of $\mathrm{T}$ cell anti-tumor function. When HIF-1 $\alpha$ is knocked out, increased fatty acid catabolism improves peroxisome proliferator-activated receptor $\alpha$ signaling in CD8+ tumor-infiltrating lymphocytes [33]. Besides, HIF-1 $\alpha$ inhibit the immunosuppressive function of Tregs, which causes the function of Tregs mainly dependent on free fatty acids in tumor microenvironment [22].
Moreover, other immune cells also affect the function of $\mathrm{T}$ cells in hypoxic microenvironment. For example, $\mathrm{B}$ cells can promote Tregs recruitment and $\mathrm{CD}^{+} \mathrm{T}$ cells exhaustion by secreting chemokines. Myeloid derived suppressor cells inhibit the metabolism of $\mathrm{T}$ cells by accumulating key amino acids, inhibit the activation of T cells by increasing PD-L1 expression, and regulate the homing of $\mathrm{T}$ cells by cleaving L-selectin. M2-type macrophages promote $\mathrm{T}$ cell nonreactivity by increasing $\mathrm{NO}$ and decreasing arginine production [53].

\section{Low glycose in the tumor environment affects $\mathrm{T}$ cell function}

Hypoxia and low glycose may send out opposite metabolic signals for $\mathrm{T}$ cells. $\mathrm{T}$ cells in the tumor microenvironment undergo glucose deprivation, leading to activated $\mathrm{T}$ cell hypo-responsiveness [45]. In $\mathrm{T}$ lymphocytes, glucose uptake and catabolism are not simply metabolic processes for nutrient utilization and energy generation. Glycolysis plays a key role in $\mathrm{T}$ cell differentiation from naïve $\mathrm{T}$ cells into tumor antigen-specific $\mathrm{T}$ effectors $[5,54]$. Thus, by creating a microenvironment condition of glucose starvation for $\mathrm{T}$ cells, cancer inhibits the differentiation and expansion of tumor-specific $\mathrm{T}$ cells exposed to tumor-associated antigens, rendering them unable to develop into tumor-specific $\mathrm{T}$ effectors. Additionally, a low-glucose microenvironment can reduce the glycolysis function of $\mathrm{T}$ cells by reducing AKT activity and induce apoptosis of tumor-infiltrating $\mathrm{T}$ cells by activating the pro-apoptotic protein family $[55,56]$. These metabolic conditions also promote $\mathrm{T}$ cells differentiation into Tregs. Besides, $\mathrm{CD}^{+}$ TILs increased FAO in the presence of both hypoglycemia and hypoxia [33]. Furthermore, oxidative neutrophils also inhibit $\mathrm{T}$ cell function under hypoglycemia [57]. Therefore, the regulation of $\mathrm{T}$ cell function requires the consideration of various metabolic factors.

\section{Metabolic intermediates in the tumor environment affect T cell function}

Metabolic intermediates produced by tumors such as tryptophan, kynurenine, and other molecules can also promote Treg differentiation and immunosuppressive function. Indo-leamine 2,3-dioxygenase (IDO) expression in tumor cells is related to tumor progression [58] and is an enzyme that degrades tryptophan [59]. Upregulation of IDO activity reduces tryptophan infiltration and induces $T$ cell apoptosis. Tumor cells must compete for energy needed for growth while diminishing Teff anti-tumor responses [8]. The lipid metabolite prostaglandin E2 (PE2) is a class of highly active inflammatory mediators that promote tumor cell survival, proliferation, invasion, metastasis, and angiogenesis. Recent studies have shown that PE2 secreted by tumor cells can stimulate the secretion of 
cancer-promoting CXCL1, interleukin-6, and granulocyte colony-stimulating factor by myeloid cells and inhibit tumor necrosis factor- $\alpha$ secretion by lipopolysaccharidestimulated myeloid cells [60].

\section{Treatments targeting $\mathrm{T}$ cell metabolism}

$T$ cells undergo metabolic reprogramming during proliferation, differentiation, and execution of effector functions. Some key signal pathways involved in metabolic reprogramming can change the energetic status. Metabolic competition in the tumor microenvironment is a new mechanism leading to strong inhibition of $\mathrm{T}$ cells. Therefore, it will be a new challenge for studies of antitumor immunotherapy to find a way are needed to develop methods for destroying the metabolism of tumor cells and while improving the ability of immune cells to obtain nutrients.

\section{Targeting $\mathrm{T}$ cell glucose metabolism}

PD-1 ligand (PD-L1) expression by tumor cells activates the $\mathrm{AKT} / \mathrm{mTOR}$ pathway to promote tumor cell glycolysis. Antibodies that block the PD-1/PD-L1 checkpoint may restore glucose levels in the tumor microenvironment, permitting $\mathrm{T}$ cell glycolysis and IFN $-\gamma$ production [61]. PD-1, which is constitutively highly expressed, is considered as a surface marker of depleted $\mathrm{CD}^{+} \mathrm{T}$ cells [62]. T cells with PD-1 activation are unable to utilize glucose and branched chain amino acids, but the ratio of FAO is increased [7]. Hypoxia stimulates the expression of PD-L1 on tumor cells to suppress the T-cell killing tumor ability [63]. Thus, PD-1/PD-L1 inhibitors can help $\mathrm{T}$ cells kill tumors by regulating $\mathrm{T}$ cell metabolism.

Similar to PD-1, lymphocyte activation gene (LAG)-3 is also an inhibitory molecule on $\mathrm{T}$ cells. It prevents excessive proliferation of naïve $\mathrm{T}$ cells by inhibiting IL7-mediated STAT5 activation. Due to increased mitochondrial content, LAG-3-deficient naïve $\mathrm{T}$ cells showed increased oxidation and glycolytic metabolism. So, targeting LAG-3 is expected to provide new ideas for anti-tumor therapy by regulating the metabolism of $\mathrm{T}$ cells [64].

Recent studies also showed that monoclonal antibodies blocking cytotoxic lymphocyte antigen 4 (CTLA-4) have been widely used in both hematologic and solid tumors [65]. CTLA-4 is constitutively expressed on the surface of chronic activated T cells. CD28 and CTLA4 share identical ligands: CD80 and CD86. Due to CTLA4 has a much higher affinity for both ligands, it can inhibit $\mathrm{T}$ cell activation by outcompeting CD28 in antigen-presenting cells such as dendritic cell and delivering inhibitory signals to cells [66]. Activated CTLA-4 competes with $\mathrm{B} 7$ ligand and recruits protein phosphatase 2 , a phosphatase that regulates the cell cycle, which inhibits the PI3K/Akt/mTOR signaling pathway and inhibits glucose uptake, thereby inhibiting $\mathrm{T}$ cell metabolism and proliferation [8].

Imatinib, a BCR-ABL kinase inhibitor, has shown opposing effects on $\mathrm{T}$ cell metabolism. It can activate $\mathrm{CD}^{+} \mathrm{T}$ cells and induce Treg cell apoptosis by downregulating IDO expression [65]. Imatinib can also decrease glucose uptake from the media by switching from glycolysis to mitochondrial glucose metabolism in BCR-ABL-positive cells [67].

As a classical regulator of glucose metabolism, metformin has direct antitumor activity and indirect CTL killing effect. By activating the LKB1-AMPK system, the mTOR pathway can be inhibited to interfere with glycolysis in the tumor, thereby inhibiting tumor growth. Besides, the regulation of metformin on PD-L1 is related to the glycosylation of PD-L1, which further promotes the activation of endoplasmic reticulum related protein degradation pathway, resulting in the downregulation of PD-L1 expression and enhancing the killing effect of CTL [68].

PIM kinase is an immune regulatory kinase that also participates in $\mathrm{T}$ cell glucose metabolism. mTORC1 activity can be enhanced by inhibiting PIM kinase, which improves the uptake of glucose by $\mathrm{T}$ cells and increases the anti-tumor function of $\mathrm{T}$ cells [69].

In addition to molecule targets, the products of some metabolic processes can also serve as therapeutic targets. For example, phosphoenolpyruvate (PEP) is an intermediate metabolite produced by glucose metabolism. The enzyme phosphoenolpyruvate carboxykinase- 1 is overexpressed during PEP production in $\mathrm{CD}^{+}$and $\mathrm{CD}^{+} \mathrm{T}$ cells, which can significantly upregulate the effector function of $\mathrm{T}$ cells and inhibit tumor growth, prolonging the survival of melanoma-bearing mice [45]. Due to the tumor microenvironment, the specific accumulation effect of some nanoparticle-mediated therapeutics can be used to improve the anti-tumor efficacy. For instance, the combination of oxaliplatin prodrug and PEGylated photosensitizer into a single nanoplatform can promote $\mathrm{T}$ cell antitumor immune response by immunogenic cell death [70]. By knocking down LDHA by RNAi nanoparticles, pyruvate metabolism is reprogrammed to reduce lactic acid production [51].

\section{Targeting T cell lipid metabolism}

Recent studies showed that metabolic reprogramming occurs in tumor cells and immune cells, intracellular cholesterol levels are significantly up-regulated in cancer cells, and their metabolites are abnormally accumulated during the development of tumor cells. However, the effect of traditional lipid metabolism drugs on $\mathrm{T}$ cells remains controversial, such as statins. It was found to have anti-tumor functions by inhibiting lipid metabolism in tumors and reducing the cholesterol level of $\mathrm{T}$ cells, 
thereby inhibiting the function of CTL cells [71]. On the other hand, it may down-regulates $\mathrm{T}$ cell expression of PD-1, 2B4, TIM-3, and LAG-3 [39].

The mevalonate kinase (MVK) metabolic pathway is involved in cholesterol synthesis. Blocking the rate-limiting enzyme of the MVK pathway in tumor cells can significantly reduce downstream metabolic production of the MVK pathway. Some studies showed that tumor cells that continuously express high MVK metabolic pathway levels can activate the immune response, revealing a new anti-tumor target for tumor immunotherapy. MVK is also crucial for T cell activation in an AKT/ mTOR signaling-dependent manner [72].

Furthermore, the ACAT-1 inhibitor avasimibe not only inhibits cholesterol esterification in tumor cells, but also increases the intracellular free cholesterol level, thereby inhibiting the proliferation and metastasis of tumor cells and enhancing the activity of $\mathrm{CD}^{+} \mathrm{T}$ cells. Avasimibe has also been used to treat cancer in tumormodel mice and showed good anti-tumor effects. A combination of avasimibe and a PD-1 antibody showed better efficacy than monotherapy in controlling tumor progression [37].

In the microenvironment with hypoglycemia and hypoxia, most of the $\mathrm{T}$ cells were inactivated with inhibitory receptors (such as PD-1 and LAG-3) up-regulation, and the free fatty acids around them were significantly increased. Fenofibrate can increase the FAO of $\mathrm{T}$ cells by activating PPAR- $\alpha$, thus reversing the inhibitory effect of $\mathrm{T}$ cells in the microenvironment [33].

\section{Targeting $\mathrm{T}$ cell amino acid metabolism}

Indoleamine-2,3-dioxygenase 1 (IDO1) catalyzes the oxidation of tryptophan into kynurenine and is partially responsible for acquired immune tolerance associated with cancer. Some studies showed that IDO expression is associated with low $\mathrm{T}$ cell infiltration and reduced survival in colorectal cancer [73]. Additionally, IDO induces Treg cell generation via an aryl hydrocarbon receptor-dependent mechanism [68].

The IDO1 small molecule inhibitor navoximod (GDC0919) is active as a combination therapy in multiple tumor models and relieves CD8+ T cell inhibition by degrading tryptophan [74]. One novel IDO inhibitor, INCB024360, showed effectiveness in mouse models by increasing $\mathrm{T}$ cell proliferation and IFN- $\gamma$ production [61]. Thus, the development of IDO inhibitors is one of the $\mathrm{T}$ cell activation modalities currently being explored.

Studies of adoptive immunotherapy showed that PD-1 expression was decreased in $\mathrm{CD}^{+} \mathrm{T}$ cells cultured

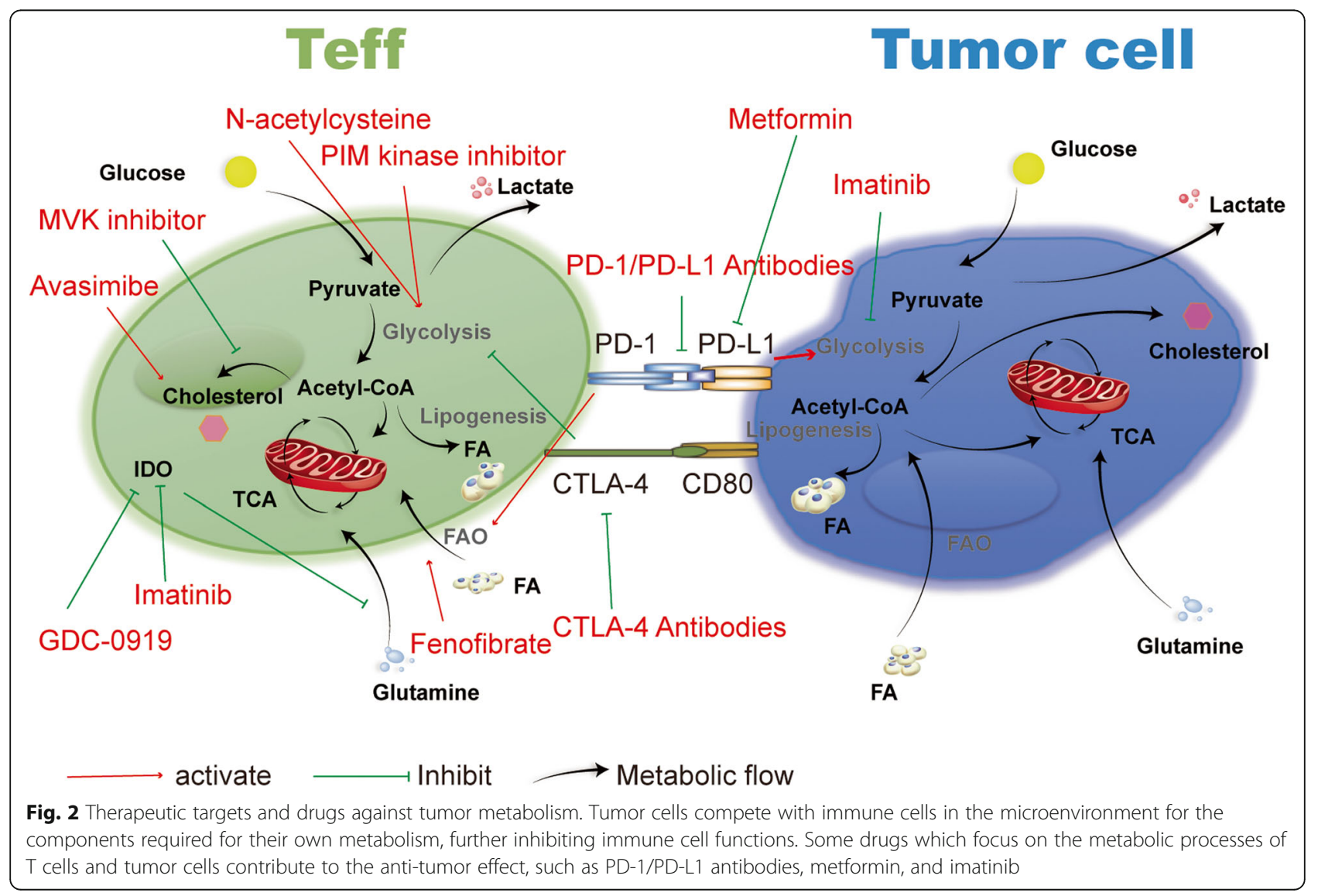


Table 1 Metabolism targeting approaches of T cell and tumor cells

\begin{tabular}{|c|c|c|c|}
\hline Name & Target & Signaling pathway & Effect \\
\hline \multirow[t]{2}{*}{ PD-1/PD-L1 Anitibodies } & PD-1/PD-L1 & $\mathrm{PI} 3 \mathrm{~K} / \mathrm{Akt} / \mathrm{mTOR}$ & Teffs: increase FAO \\
\hline & & & Tumor: inhibit glycolysis \\
\hline CTLA-4 Antibodies & CTLA-4 & PI3K/Akt/mTOR & Teffs: inhibit glucose uptake \\
\hline \multirow[t]{3}{*}{ Imatinib } & BCR-ABL kinase/IDO & BCR/ABL IDO & Teffs: activate \\
\hline & & & Treg: apoptosis \\
\hline & & & $\begin{array}{l}\text { Tumor: switching from glycolysis } \\
\text { to OXPHOS }\end{array}$ \\
\hline Metformin & PD-L1 & LKB1-AMPK system mTOR & $\begin{array}{l}\text { Tumor: down-regulate PD-L1 } \\
\text { expression }\end{array}$ \\
\hline PIM kinase inhibitor & PIM kinase & mTORC1 & Teffs: increase glucose uptake \\
\hline $\begin{array}{l}\text { Enzyme phosphoenolpyruvate } \\
\text { carboxykinase-1 }\end{array}$ & Phosphoenolpyruvate & $\begin{array}{l}\text { Sarco/ER } \mathrm{Ca}(2+) \text {-ATPase } \\
\text { (SERCA) activity }\end{array}$ & $\begin{array}{l}\text { Teffs: upregulate the effector } \\
\text { function }\end{array}$ \\
\hline \multirow[t]{2}{*}{ MVK inhibitor } & MVK & PI3K/Akt/mTOR & Teffs: promote activation \\
\hline & & & Tumor: inhibit \\
\hline \multirow[t]{2}{*}{ Avasimibe } & ACAT-1 & Cholesterol esterification & Teffs: activate \\
\hline & & & $\begin{array}{l}\text { Tumor: inhibit the proliferation and } \\
\text { metastasis }\end{array}$ \\
\hline GDC-0919 & IDO1 & tryptophan & Teffs: relieves CD8+ T cell inhibition \\
\hline INCB024360 & IDO & tryptophan & $\begin{array}{l}\text { Teffs: increase proliferation and IFN- } \gamma \\
\text { production }\end{array}$ \\
\hline $\mathrm{N}$-acetylcysteine & FOXO1 & PI3K/Akt/mTOR & $\begin{array}{l}\text { Teffs: affect granzyme B secretion } \\
\text { and PD-1 expression }\end{array}$ \\
\hline
\end{tabular}

under glutamine-limited conditions, while Ki67 and prosurvival factor expression was increased. Therefore, a novel approach for culturing $\mathrm{CD}^{+} \mathrm{T}$ cells under glutamine restriction may be a promising strategy for improving adoptive immunotherapy [75]. Similarly, Nacetylcysteine can inhibit FOXO1 expression by activating the PI3K/AKT signaling pathway, thus affecting granzyme $B$ secretion and PD-1 expression to further increase the anti-tumor ability of $\mathrm{T}$ cells amplified in vitro [76].

\section{Conclusion}

$\mathrm{T}$ cell metabolism can be altered to perform different cellular functions. To fulfill the rapid growth and produce energy, metabolism in $\mathrm{T}$ cells is switched from OXPHOS to glycolysis and glutamine metabolism to support cell growth and proliferation as well as lipid and nucleotide synthesis. Therefore, determining the reasons for $\mathrm{T}$ cell differentiation in the tumor microenvironment is helpful for clarifying the metabolic requirements and regulatory modes of different $\mathrm{T}$ cell subtypes. Metabolic reactions in tumor cells and immune cells are regulated by nutrients and metabolites in the microenvironment. By studying glucose, amino acid, and lipid metabolism pathways in tumor and $\mathrm{T}$ cells, novel anti-tumor therapeutic targets may be revealed. (Fig. 2, Table 1) However, balancing the inhibition of tumors and maintenance of immune cell activity remains challenging. In addition to the roles of checkpoint inhibitors, which directly affect tumor cells, the metabolism of immune cells requires further analysis. To evaluate specific metabolic pathways, metabolites and metabolic enzymes that regulate $\mathrm{T}$ cell metabolism to enhance the ability of $\mathrm{T}$ cells to kill tumors and exert antitumor effects on tumor cell metabolism require additional analysis. Due to the competition of nutrients between tumor and $\mathrm{T}$ cells, the metabolic adaptation of cells to microenvironment is the key to maintain the cell function. Besides, since the interaction between immune cells can affect the tumor suppressive microenvironment, future research may focus on the mechanism of same metabolic molecule in different cells. Above all, the discovery of drugs that can both enhance anti-tumor immunity and directly kill tumors, such as imatinib and ACAT-1 inhibitors, is the focus of future drug development.

\footnotetext{
Abbreviations

ACAT: Acyl-CoA acyltransferase; ACLY: ATP citrate lyase; CBP: Cholesterol biosynthesis pathway; CTL: Cytotoxic lymphocyte; CTLA-4: Cytotoxic lymphocyte antigen 4; FAO: Fatty acid oxidation; FAS: Fatty acid synthesis; FASN: Fatty acid synthase; IDO: Indoleamine 2,3-dioxygenase; LXR: Liver X receptor; MVK: Mevalonate; OXPHOS: Oxidative phosphorylation; PD-1: Death protein 1; PD-L1: Death protein 1 ligand; PEP: Phosphoenolpyruvate; PPAR: Peroxisome proliferator-activated receptors; SERBP: Sterol regulatory element-binding protein; TCR: T-cell antigen receptor; Teff: Effector T cell; TILs: Tumor infiltrating lymphocytes; Tregs: Regulatory T cells
}

Acknowledgements Not applicable. 


\section{Authors' contributions}

ZPY and LB carried out the primary literature search, drafted and revised the manuscript, and participated in discussions. WL, TLZ and HMT helped modify the manuscript. JWC carried out the design of the research and literature analysis, drafted and revised the manuscript, and participated in discussions. All authors read and approved the final manuscript.

\section{Funding}

This work was supported by the National Natural Science Foundation of China (Grant 81672275 and 81874052 to JW.C): the Project of Health and Family Planning Commssion of Jilin Province (Grant 2017 J064 to JW.C); the Project of Jilin Provincial Department of Education (Grant JJKH20190020KJ to JW.C); the Project of Department of Finance of Jilin Province (Grant 2018SCZWSZX-010 to JW.C); Industrial Research and Development Project of Development and Reform Commission of Jilin Province (Grant 2017C022 to JW.C); Key Laboratory Construction Project of Science and Technology Department (Grant 20170622011JC to JW.C); Project of Department of Science and Technology of Jilin Province (Grants 20180101009JC and 20190303146SF to JW.C); Outstanding Talent Cultivation Program for Doctoral Students in Norman Bethune Health Science Center of Jilin University (Grant to L.B), and Science Development Project of Jilin Province (Grant 20150520046JH to HM.T).

\section{Availability of data and materials}

Not applicable

\section{Ethics approval and consent to participate}

Not applicable

\section{Consent for publication}

Not applicable

\section{Competing interests}

The authors declare that they have no competing interests.

Received: 30 May 2019 Accepted: 3 September 2019

Published online: 13 September 2019

\section{References}

1. Fernández-Ramos AA, Marchetti-Laurent C, Poindessous V, Antonio S, Petitgas C, Ceballos-Picot I, et al. A comprehensive characterization of the impact of mycophenolic acid on the metabolism of Jurkat T cells. Sci Rep. 2017;7:10550.

2. Phan LM, Yeung SC, Lee MH. Cancer metabolic reprogramming: importance, main features, and potentials for precise targeted anti-cancer therapies. Cancer Biol Med. 2014;11:1-19.

3. Hope HC, Salmond RJ. Targeting the tumor microenvironment and T cell metabolism for effective cancer immunotherapy. Eur J Immunol. 2019;49(8): 1147-52. https://doi.org/10.1002/eji.201848058 Epub 2019 Jul 9.

4. Kouidhi S, Elgaaied AB, Chouaib S. Impact of metabolism on T-cell differentiation and function and cross talk with tumor microenvironment. Front Immunol. 2017:8:270

5. Chang $\mathrm{CH}$, Curtis JD, Maggi LB, Faubert B, Aillarino AV, O'Sullivan D, et al. Posttranscriptional control of $T$ cell effector function by aerobic glycolysis. Cell. 2013;153:1239-51.

6. Ogando J, Sáez ME, Santos J, Nuevo-Tapioles C, Gut M, Esteve-Codina A, et al. PD-1 signaling affects cristae morphology and leads to mitochondrial dysfunction in human CD8+ T lymphocytes. J Immunother Cancer. 2019; 7(1):151. https://doi.org/10.1186/s40425-019-0628-7.

7. Patsoukis N, Bardhan K, Chatterjee P, Sari D, Liu B, Bell LN, et al. PD-1 alters T-cell metabolic reprogramming by inhibiting glycolysis and promoting lipolysis and fatty acid oxidation. Nat Commun. 2015;6:6692.

8. Ho PC, Liu PS. Metabolic communication in tumors: a new layer of immunoregulation for immune evasion. J Immunother Cancer. 2016;4:4.

9. Yu X, Teng XL, Wang F, Zheng Y, Qu G, Zhou Y, et al. Metabolic control of regulatory $T$ cell stability and function by TRAF3IP3 at the lysosome. J Exp Med. 2018;215(9):2463-76. https://doi.org/10.1084/jem. 20180397 Epub 2018 Aug 16.

10. Fernandez-Ramos AA, Poindessous V, Marchetti-Laurent C, Pallet N, Loriot MA. The effect of immunosuppressive molecules on T-cell metabolic reprogramming. Biochimie. 2016;127:23-36.
11. Slack M, Wang T, Wang R. T cell metabolic reprogramming and plasticity. Mol Immunol. 2015;68:507-12.

12. Jiang S, Yan W. T-cell immunometabolism against cancer. Cancer Lett. 2016; 382:255-8.

13. Simon-Molas H, Arnedo-Pac C, Fontova P, Vidal-Alabro A, Castano E, Rodriguez-Garcia A, et al. PI3K-Akt signaling controls PFKFB3 expression during human T-lymphocyte activation. Mol Cell Biochem. 2018;448:187-97.

14. Hu Z, Qu G, Yu X, Jiang H, Teng XL, Ding L, et al. Acylglycerol Kinase Maintains Metabolic State and Immune Responses of CD8+ T Cells. Cell Metab. 2019;30(2):290-302. https://doi.org/10.1016/j.cmet.2019.05.016 Epub 2019 Jun 13. e5.

15. Zhou X, Yu J, Cheng X, Zhao B, Manyam GC, Zhang L, et al. The deubiquitinase Otub1 controls the activation of CD8+ T cells and NK cells by regulating IL-15-mediated priming. Nat Immunol. 2019;20(7):879-89. https://doi.org/10.1038/s41590-019-0405-2 Epub 2019 Jun 10.

16. Wang R, Dillon C, Shi L, et al. The transcription factor Myc controls metabolic reprogramming upon T lymphocyte activation. Immunity. 2011;35:0-882.

17. Zhang T, Zhang Z, Li F, Ping Y, Qin G, Zhang C, et al. miR-143 Regulates Memory T Cell Differentiation by Reprogramming T Cell Metabolism. J Immunol. 2018;201(7):2165-75. https://doi.org/10.4049/jimmunol.1800230 Epub 2018 Aug 27.

18. DeVorkin L, Pavey N, Carleton G, Comber A, Ho C, Lim J, et al. Autophagy Regulation of Metabolism Is Required for CD8+ T Cell Anti-tumor Immunity. Cell Rep. 2019;27(2):502-13. https://doi.org/10.1016/j.celrep.2019.03.037 e5.

19. Gemta LF, Siska PJ, Nelson ME, Gao X, Liu X, Locasale JW, et al. Impaired enolase 1 glycolytic activity restrains effector functions of tumor-infiltrating CD8+ T cells. Sci Immunol. 2019;4(31). https://doi.org/10.1126/sciimmunol. aap9520.

20. Qiu J, Villa M, Sanin DE, Buck MD, O'Sullivan D, Ching R, et al. Acetate Promotes T Cell Effector Function during Glucose Restriction. Cell Rep. 2019; 27(7):2063-74. https://doi.org/10.1016/j.celrep.2019.04.022 e5.

21. Prado-Garcia H, Romero-Garcia S, Castro-Flores DA, Rumbo-Nava U, et al. Deficient glucose uptake is linked to impaired Glut1 expression upon CD3/ CD28 stimulation in memory T cells from pleural effusions secondary to lung cancer. Scand J Immunol. 2019;90(3):e12802. https://doi.org/10.1111/sji. 12802 Epub 2019 Jul 21.

22. Miska J, Lee-Chang C, Rashidi A, Muroski ME, Chang AL, Lopez-Rosas A, et al. HIFla Is a Metabolic Switch between Glycolytic-Driven Migration and Oxidative Phosphorylation-Driven Immunosuppression of Tregs in Glioblastoma. Cell Rep. 2019;27(1):226-37. https://doi.org/10.1016/j.celrep.2019.03.029 e4.

23. O'Sullivan D, van der Windt GJ, Huang SC, Curtis JD, Chang CH, Buck MD, ,et al Memory CD8(+) T cells use cell-intrinsic lipolysis to support the metabolic programming necessary for development. Immunity. 2014;41(1): 75-88. doi: https://doi.org/10.1016/j.immuni.2014.06.005. Epub 2014 Jul 4.

24. Maciver NJ, Michalek RD, Rathmell JC. Metabolic regulation of T ymphocytes. Annu Rev Immunol. 2013;31:259-83.

25. Cheng C, Geng F, Cheng X, Guo D. Lipid metabolism reprogramming and its potential targets in cancer. Cancer Commun (Lond). 2018;38(1):27. https://doi.org/10.1186/s40880-018-0301-4.

26. Xu T, Stewart KM, Wang X, Liu K, Xie M, Ryu JK, et al. Metabolic control of TH17 and induced Treg cell balance by an epigenetic mechanism. Nature. 2017;548:228-33.

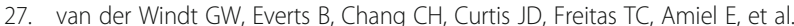
Mitochondrial respiratory capacity is a critical regulator of CD8+ T cell memory development. Immunity. 2012;36(1):68-78.

28. Liu C, Chikina M, Deshpande R, Menk AV, Wang T, Tabib T, et al. Treg Cells Promote the SREBP1-Dependent Metabolic Fitness of Tumor-Promoting Macrophages via Repression of CD8+ T Cell-Derived Interferon- $\gamma$. Immunity. 2019; 51(2):381-97. https://doi.org/10.1016/.jimmuni.2019.06.017 Epub 2019 Jul 23. e6.

29. Chakraborty P, Vaena SG, Thyagarajan K, Chatterjee S, Al-Khami A, Selvam SP, et al. Pro-Survival Lipid Sphingosine-1-Phosphate Metabolically Programs T Cells to Limit Anti-tumor Activity. Cell Rep. 2019;28(7):1879-93. https://doi. org/10.1016/j.celrep.2019.07.044 e7.

30. Bensinger SJ, Bradley MN, Joseph SB, Zelcer N, Janssen EM, Hausner MA, et al. LXR signaling couples sterol metabolism to proliferation in the acquired immune response. Cell. 2008;134(1):97-111. https://doi.org/10.1016/j.cell. 2008.04.052.

31. Kidani $Y$, Elsaesser $H$, Hock MB, Vergnes L, Williams KJ, Argus JP, et al. Sterol regulatory element-binding proteins are essential for the metabolic programming of effector T cells and adaptive immunity. Nat Immunol. 2013;14(5):489-99. https://doi.org/10.1038/ni.2570 Epub 2013 Apr 7. 
32. Perucha E, Melchiotti R, Bibby JA, Wu W, Frederiksen KS, Roberts CA, et al. The cholesterol biosynthesis pathway regulates IL-10 expression in human Th1 cells. Nat Commun. 2019;10(1):498. https://doi.org/10.1038/s41467-019-08332-9.

33. Zhang Y, Kurupati R, Liu L, Zhou XY, Zhang G, Hudaihed A, et al. Enhancing CD8+ $T$ cell fatty acid catabolism within a metabolically challenging tumor microenvironment increases the efficacy of melanoma immunotherapy. Cancer Cell. 2017;32:377-91.

34. Bailis W, Shyer JA, Chiorazzi M, Flavell RA. No oxygen? No glucose? No problem: fatty acid catabolism enhances effector CD8+ TILs. Cancer Cell. 2017;32:280-1.

35. Zhang $L$, Romero P. Metabolic control of CD8(+) T cell fate decisions and antitumor immunity. Trends Mol Med. 2018;24:30-48.

36. Bai R, Bai L, Li W, Cui JW. Research progress on anti-tumor therapy targeting cholesterol metabolism. J Nutr Oncol. 2017;2:4

37. Yang W, Bai $Y$, Xiong $Y$, Zhang J, Chen S, Zheng $X$, et al. Potentiating the antitumour response of CD8(+) T cells by modulating cholesterol metabolism. Nature. 2016;531:651-5

38. Ma X, Bi E, Huang C, Lu Y, Xue G, Guo X, et al. Cholesterol negatively regulates IL-9-producing CD8+ T cell differentiation and antitumor activity. J Exp Med. 2018;215(6):1555-69. https://doi.org/10.1084/jem.20171576 Epub 2018 May 9

39. Ma X, Bi E, Lu Y, Su P, Huang C, Liu L, et al. Cholesterol Induces CD8+ T Cell Exhaustion in the Tumor Microenvironment. Cell Metab. 2019;30(1):143-56. https://doi.org/10.1016/j.cmet.2019.04.002 Epub 2019 Apr 25. e5.

40. McKinney EF, Smith KGC. Metabolic exhaustion in infection, cancer and autoimmunity. Nat Immunol. 2018;19(3):213-21. https://doi.org/10.1038/ s41590-018-0045-y Epub 2018 Feb 5.

41. Patel $\mathrm{CH}$, Powell JD. Targeting T cell metabolism to regulate T cell activation, differentiation and function in disease. Curr Opin Immunol. 2017:46:82-8.

42. Johnson MO, Wolf MM, Madden MZ, Andrejeva G, Sugiura A, Contreras DC, et al. Distinct Regulation of Th17 and Th1 Cell Differentiation by Glutaminase-Dependent Metabolism. Cell. 2018;175(7):1780-95. https://doi org/10.1016/j.cell.2018.10.001 Epub 2018 Nov 1. e19.

43. Song M, Sandoval TA, Chae CS, Chopra S, Tan C, Rutkowski MR, et al. IRE1a-XBP1 controls $T$ cell function in ovarian cancer by regulating mitochondrial activity. Nature. 2018;562(7727):423-8. https:/doi.org/10.1038/s41586-018-0597-x Epub 2018 Oct 10.

44. Chang CH, Qiu J, O'Sullivan D, Buck MD, Noguchi T, Curtis JD, et al. Metabolic competition in the tumor microenvironment is a driver of cancer progression. Cell. 2015;162:1229-41.

45. Ho PC, Bihuniak JD, Macintyre AN, Staron M, Liu X, Amezquita R, et al. Phosphoenolpyruvate is a metabolic checkpoint of anti-tumor $T$ cell responses. Cell. 2015;162:1217-28.

46. Seema G, Amrita R, Dwarakanath BS. Metabolic cooperation and competition in the tumor microenvironment: implications for therapy. Front Oncol. 2017:7:68.

47. Waickman AT, Powell JD. mTOR, metabolism, and the regulation of T-cell differentiation and function. Immunol Rev. 2012;249:43-58.

48. Duvel K, Yecies JS, Raman P, Lipovsky Al, Souza AL, Triantafellow E, et al. Activation of a metabolic gene regulatory network downstream of mTOR complex 1. Mol Cell. 2010;39:171-83.

49. Fischer K, Hoffmann P, Voelkl S, Meidenbauer N, Ammer J, Edinger M, et al. Inhibitory effect of tumor cell-derived lactic acid on human T cells. Blood. 2007;109:3812-9.

50. Påhlman C, Qi Z, Murray CM, Ferguson D, Bundick RV, Donald DK, et al. Immunosuppressive properties of a series of novel inhibitors of the monocarboxylate transporter MCT-1. Transpl Int. 2013;26:22-9.

51. Zhang YX, Zhao YY, Shen J, Sun X, Liu Y, Liu H, et al. Nanoenabled modulation of acidic tumor microenvironment reverses Anergy of infiltrating T cells and potentiates anti-PD-1 therapy. Nano Lett. 2019;19(5): 2774-83. https://doi.org/10.1021/acs.nanolett.8b04296 Epub 2019 Apr 5.

52. Gropper Y, Feferman T, Shalit T, Salame TM, Porat Z, Shakhar G. Culturing CTLs under hypoxic conditions enhances their cytolysis and improves their anti-tumor function. Cell Rep. 2017;20:2547-55.

53. Daniel SK, Sullivan KM, Labadie KP, Pillarisetty VG. Hypoxia as a barrier to immunotherapy in pancreatic adenocarcinoma. Clin Transl Med. 2019;8(1): 10. https://doi.org/10.1186/s40169-019-0226-9.

54. Michalek RD, Gerriets VA, Jacobs SR, Macintyre AN, Maclver NJ, Mason EF, et al. Cutting edge: distinct glycolytic and lipid oxidative metabolic programs are essential for effector and regulatory CD4+ T cell subsets. J Immunol. 2011;186:3299-303.

55. Vander Heiden MG, Plas DR, Rathmell JC, Fox CJ, Harris MH, Thompson CB. Growth factors can influence cell growth and survival through effects on glucose metabolism. Mol Cell Biol. 2001;21(17):5899-912.
56. Buchakjian MR, Kornbluth S. The engine driving the ship: metabolic steering of cell proliferation and death. Nat Rev Mol Cell Biol. 2010;11:715-27.

57. Rice CM, Davies LC, Subleski JJ, Maio N, Gonzalez-Cotto M, Andrews C, et al. Tumour-elicited neutrophils engage mitochondrial metabolism to circumvent nutrient limitations and maintain immune suppression. Nat Commun. 2018;9(1):5099. https://doi.org/10.1038/s41467-018-07505-2.

58. Munn DH, Sharma MD, Baban B, Harding HP, Zhang Y, Ron D, et al. GCN2 kinase in T cells mediates proliferative arrest and anergy induction in response to indoleamine 2,3-dioxygenase. Immunity. 2005;22:633-42.

59. Opitz CA, Litzenburger UM, Sahm F, Ott M, Tritschler I, Trump S, et al. An endogenous tumour-promoting ligand of the human aryl hydrocarbon receptor. Nature. 2011;478:197-203

60. Ma X, Aoki T, Tsuruyama T, Narumiya S. Definition of prostaglandin E2-EP2 signals in the colon tumor microenvironment that amplify inflammation and tumor growth. Cancer Res. 2015;75:2822-32.

61. Staron M, Gray S, Marshall H, Parish IA, Chen JH, Perry CJ, et al. The transcription factor FoxO1 sustains expression of the inhibitory receptor PD1 and survival of antiviral CD8+ T cells during chronic infection. Immunity. 2014;41:802-14.

62. Huang A, Peng D, Guo H, Ben Y, Zuo X, Wu F, et al. A human programmed death-ligand 1-expressing mouse tumor model for evaluating the therapeutic efficacy of anti-human PD-L1 antibodies. Sci Rep. 2017:7:42687.

63. McDonald PC, Chafe SC, Dedhar S. Overcoming hypoxia-mediated tumor progression: combinatorial approaches targeting $\mathrm{pH}$ regulation, angiogenesis and immune dysfunction. Front Cell Dev Biol. 2016;4:27.

64. Previte DM, Martins CP, O'Connor EC, Marre ML, Coudriet GM, Beck NW, et al. Lymphocyte Activation Gene-3 Maintains Mitochondrial and Metabolic Quiescence in Naive CD4+ T Cells. Cell Rep. 2019;27(1):129-41. https://doi. org/10.1016/j.celrep.2019.03.004 e4.

65. Beckermann KE, Dudzinski SO, Rathmell JC. Dysfunctional T cell metabolism in the tumor microenvironment. Cytokine Growth Factor Rev. 2017;35:7-14.

66. Pardoll DM. The blockade of immune checkpoints in cancer immunotherapy. Nat Rev Cancer. 2012:12(4):252-64.

67. Gottschalk S. Imatinib (STI571)-mediated changes in glucose metabolism in human leukemia BCR-ABL-positive cells. Clin Cancer Res. 2004;10:6661-8.

68. Cha JH, Yang WH, Xia W, Wei Y, Chan LC, Lim SO, et al. Metformin promotes antitumor immunity via endoplasmic-reticulum-associated degradation of PD-L1. Mol Cell. 2018;71:606-20.

69. Chatterjee S, Chakraborty P, Daenthanasanmak A, lamsawat S, Andrejeva G, Luevano LA, et al. Targeting PIM kinase with PD1 inhibition improves immunotherapeutic antitumor T-cell response. Clin Cancer Res. 2019;25: 1036-49.

70. Zhou F, Feng B, Yu H, Wang D, Wang T, Ma Y, et al. Tumor microenvironment-Activatable prodrug vesicles for Nanoenabled Cancer Chemoimmunotherapy combining immunogenic cell death induction and CD47 blockade. Adv Mater. 2019;31(14):e1805888. https://doi.org/10.1002/ adma.201805888 Epub 2019 Feb 14.

71. Frostegård J, Zhang Y, Sun J, Yan K, Liu A. Oxidized low-density lipoprotein (OxLDL)-treated dendritic cells promote activation of T cells in human atherosclerotic plaque and blood, which is repressed by statins: microRNA let-7c is integral to the effect. J Am Heart Assoc. 2016;5:e003976.

72. Thurnher M, Gruenbacher G. T Iymphocyte regulation by mevalonate metabolism. Sci Signal. 2015;8:re4

73. Muller AJ, Manfredi MG, Zakharia Y, Prendergast GC. Inhibiting IDO pathways to treat cancer: lessons from the ECHO-301 trial and beyond. Semin Immunopathol. 2019;41:41-8.

74. Nayak-Kapoor A, Hao Z, Sadek R, Dobbins R, Marshall L, Vahanian NN, et al. Phase la study of the indoleamine 2,3-dioxygenase 1 (IDO1) inhibitor navoximod (GDC-0919) in patients with recurrent advanced solid tumors. J Immunother Cancer. 2018;6:61.

75. Nabe S, Yamada T, Suzuki J, Toriyama K, Yasuoka T, Kuwahara M, et al. Reinforce the antitumor activity of CD8+ T cells via glutamine restriction. Cancer Sci. 2018;109:3737-50.

76. Scheffel MJ, Scurti G, Wyatt MM, Garrett-Mayer E, Paulos CM, Nishimura MI, et al. N-acetyl cysteine protects anti-melanoma cytotoxic T cells from exhaustion induced by rapid expansion via the downmodulation of Foxo1 in an Aktdependent manner. Cancer Immunol Immunother. 2018;67:691-702.

\section{Publisher's Note}

Springer Nature remains neutral with regard to jurisdictional claims in published maps and institutional affiliations. 